TRANSACTIONS OF THE

AMERICAN MATHEMATICAL SOCIETY

Volume 185, November 1973

\title{
THE LAW OF THE ITERATED LOGARITHM FOR BROWNIAN MOTION IN A BANACH SPACE
}

\author{
BY \\ J. KUELBS(1) AND R. LEPAGE
}

\begin{abstract}
Strassen's version of the law of the iterated logarithm is proved for Brownian motion in a real separable Banach space. We apply this result to obtain the law of the iterated logarithm for a sequence of independent Gaussian random variables with values in a Banach space and to obtain Strassen's result.
\end{abstract}

Introduction. Let $H$ denote a real separable Hilbert space with norm $\|\cdot\|_{H}$ and assume $\|\cdot\|_{B}$ is a measurable norm on $H$ in the sense of [2]. Then there exists a constant $M>0$ such that $\|x\|_{B} \leq M\|x\|_{H}$ for all $x \in H$, and if $B$ is the completion of $H$ in $\|\cdot\|_{B}$ it follows that $B$ is a real separable Banach space. We will view $H$ as a subspace of $B$ and since $\|\cdot\|_{B}$ is weaker than $\|\cdot\|_{H}$ on $H$ it follows that $B^{*}$, the topological dual of $B$, can be continuously injected into $H^{*}$, the topological dual of $H$. We call $(H, B)$ an abstract Wiener space.

For $t>0$, let $m_{t}$ denote the canonical Gaussian cylinder set measure on $H$ with variance parameter $t$ and let $\mu_{t}(t>0)$ denote the Borel probability measure on $B$ induced by $m_{t}(t>0)$. We call $\mu_{t}$ the Wiener measure on $B$ generated by $H$ with variance parameter $t$.

Let $\Omega_{B}$ denote the space of continuous functions $w$ from $[0, \infty)$ into $B$ such that $w(0)=0$, and let $\exists$ be the $\sigma$-field of $\Omega_{B}$ generated by the functions $w \rightarrow w(t)$. Then there is a unique probability measure $P$ on 9 such that if $0=t_{0}<t_{1}$ $<\cdots<t_{n}$ then $w\left(t_{j}\right)-w\left(t_{j-1}\right)(j=1, \ldots, n)$ are independent and $w\left(t_{j}\right)$ - $w\left(t_{j-1}\right)$ has distribution $\mu_{t_{j-1}-1}$ on $B$. In particular, the stochastic process $W_{t}$ defined on $\left(\Omega_{B}, \Im, P\right)$ by $W_{t}(w)=w(t)$ has stationary independent Gaussian increments with transition probabilities $P_{t}(x, A)=\mu((A-x) / \sqrt{t})$ for $t>0$. We call it Brownian motion in $B$. For a more detailed discussion see [2].

It is known from [2] that if $B$ is an arbitrary real separable Banach space, then there exists a dense subset $H$ of $B$ which is a real separable Hilbert space and the given norm on $B$ is a measurable norm on $H$. Hence any real separable Banach space can be used in the setup we described above. We also know from [5] or from [6] and [1] that if $\mu$ is any mean zero Gaussian probability measure on the Borel subsets of a real separable Banach space $B$, then there exists a real separable Hilbert space $H$ which is a subset of $B$, the given norm on $B$ is a

Received by the editors June 21, 1972. $28 \mathrm{~A} 40$.

AMS (MOS) subject classifications (1970). Primary 60G15, 60G17, 60B05, 60J65; Secondary

Key words and phrases. Abstract Wiener spaces, measurable norm, Gaussian measures, Strassen law of the iterated logarithm, Brownian motion in a Banach space.

(1) Supported in part by NSF Grant GP 18759. 
measurable norm on $H, \mu(M)=1(M$ is the closure of $H$ in $B$ ), and $\mu$ is the Wiener measure on $M$ generated by $H$ with variance parameter 1 . Furthermore, $H$ is unique as a subset of $B$ since it is precisely the set of vectors such that $\mu$ translated by such a vector yields a measure equivalent to $\mu$. However, the main point to be realized is that given any real separable Banach space $B$, or $B$ and a mean zero Gaussian measure $\mu$ on $B$, we can construct a Brownian motion on $B$ as indicated above. Further, in the case $\mu$ is given on $B$ we see that $\mu=\mu_{1}$ and that if $M$ is a proper subspace of $B$ then our Brownian motion is, with probability one, in the closed subspace $M$ satisfying $\mu_{1}(M)=1$.

Let $C_{B}$ denote the continuous functions on $[0,1]$ into $B$ which vanish at zero. Then $C_{B}$ is a Banach space in the norm $\|f\|_{C_{B}}=\sup _{0 \leq t \leq 1}\|f(t)\|_{B}$.

Lemma 1. (a) If $B$ is a real separable Banach space, then $C_{B}$ is a real separable Banach space in the norm $\|\cdot\|_{C_{B}}$.

(b) The minimal sigma-algebra $B$ making the mappings $f \rightarrow f(t)$ measurable consists of the Borel subsets of $C_{B}$.

(c) Brownian motion on $B$ induces a probability measure $P$ on $\left(C_{B}, \mathcal{B}\right)$ which is a mean zero Gaussian measure, i.e. every linear functional in $C_{B}^{*}$ has a Gaussian distribution with mean zero.

Proof. (a) Let $t_{j}=j / 2^{N}, j=0,1, \ldots, 2^{N}$. Let $\left\{x_{n}\right\}$ be a dense subset of $B$. Let $S_{N}$ denote the subset of $C_{B}$ consisting of functions which are linear on each of the subintervals $\left[t_{j-1}, t_{j}\right]$ with values at $t_{j}$ in $\left\{x_{n}\right\}$. Then $\cup_{N=1}^{\infty} S_{N}$ is a countable dense subset of $C_{B}$.

(b) Since $C_{B}$ is separable it suffices to prove that if $f_{0} \in C_{B}$ and $\epsilon>0$, then $U=\left\{f:\left\|f-f_{0}\right\|_{C_{B}} \leq \epsilon\right\}$ is a set in $\mathcal{B}$. Let $I_{N}=\left\{f: \sup _{1 \leq j \leq 2^{N}}\left\|f\left(t_{j}\right)-f_{0}\left(t_{j}\right)\right\|_{C_{B}}\right.$ $\leq \epsilon\}$ for $N=1,2, \ldots$ and $\left\{t_{j}\right\}$ as in (a). Then $U=\cap_{N=1}^{\infty} I_{N}$.

(c) $P$ is the probability measure on $\left(C_{B}, B\right)$ such that if $0=t_{0}<t_{1}<\cdots<t_{n}$ $\leq 1$ then $f\left(t_{j}\right)-f\left(t_{j-1}\right)(j=1, \ldots, n)$ are independent and $f\left(t_{j}\right)-f\left(t_{j-1}\right)$ has distribution $\mu_{t,-t_{-1}}$ on $B$. We now must show $P$ is a mean zero Gaussian measure on $C_{B}$. Let $f^{*} \in C_{B}^{*}$ and let $X_{1}, \ldots, X_{n}$ be independent random variables with values in $C_{B}$ and the same distribution as $P$. Then $X_{1}+\cdots+X_{n} / \sqrt{n}$ has distribution $P$ since for each $t \in[0,1]$ the law of the map $f \rightarrow f(t)$ is the convolution of $\mu_{t / n} n$ times yielding $\mu_{t}$. Hence the distribution of $f^{*}$ has the same distribution as

$$
f^{*}\left(X_{1}+\cdots+X_{n} / \sqrt{n}\right)=f^{*}\left(X_{1}\right)+\cdots+f^{*}\left(X_{n}\right) / \sqrt{n}
$$

and we see by $\left[4\right.$, p. 166] that $f^{*}$ is strictly stable with characteristic exponent 2 and this implies $f^{*}$ has a Gaussian distribution with mean zero.

Our main result is a law of the iterated logarithm for Brownian motion in a Banach space as described prior to Lemma 1. This may be regarded as a general synthesis of the two log log law which follows.

I. (Strassen [10]). Let $\Omega_{k}$ denote the set of continuous maps from $[0, \infty)$ into 
real $k$-dimensional space $\left(\mathbf{R}^{k}\right)$ which vanish at zero, and let $C_{k}$ denote the space of continuous maps vanishing at zero and mapping $[0,1]$ into $\mathbf{R}^{k}$ endowed with the supremum of the Euclidean norm for $\mathbf{R}^{k}$. If $W(t)=\left(W_{1}(t), \ldots, W_{k}(t)\right)$, $0 \leq t<\infty$, is a version of the $k$-dimensional Brownian motion with sample paths in $\Omega_{k}$, then the sequence of random functions

$$
\zeta_{n}(t)=W(n t) /(2 n \log \log n)^{1 / 2} \quad(0 \leq t \leq 1, n \geq 3)
$$

satisfies the following $\log \log$ law:

$$
\left\{\zeta_{n}, n \geq 3\right\} \subseteq C_{k} \text { and with probability one converges in } C_{k}
$$

to a compact set $K_{k}$ of $C_{k}$ and clusters at every point of $K_{k}$.

Here $K_{k}$ denotes those $f=\left(f_{1}, \ldots, f_{k}\right) \in C_{k}$ such that $f$ is coordinatewise absolutely continuous with respect to Lebesgue measure on $[0,1]$, and satisfies $\sum_{i=1}^{k} \int_{0}^{1}\left[d f_{i}(s) / d s\right]^{2} d s \leq 1$. By saying $\left\{\zeta_{n}: n \geq 3\right\}$ converges to $K_{k}$ we mean that for every $\epsilon>0$ the sequence is eventually within an $\epsilon$-neighborhood of $K_{k}$ and since $K_{k}$ is compact this implies that with probability one $\left\{\zeta_{n}: n \geq 3\right\}$ is relatively norm compact in $C_{k}$.

II. (LePage [8]). Suppose $B$ is a real separable Banach space and $\mu$ is a mean zero Gaussian measure on the Borel subsets of $B$. If $X_{1}, X_{2}, \ldots$ are independent identically distributed $B$-valued random vectors with distribution $\mu$, then the sequence

$$
\xi_{n}=X_{1}+\cdots+X_{n} /(2 n \log \log n)^{1 / 2} \quad(n \geq 3)
$$

almost surely converges in $B$-norm to a closed set $K \subseteq B$ and clusters at every point of $K$, where $K$ is the unit ball of the reproducing kernel Hilbert space defined on $B^{*} \times B^{*}$ by $\mu$.

The set $K_{k}$ of Strassen's result may be identified as the unit ball.of the reproducing kernel Hilbert space of the kernel defined for $0 \leq s, t \leq 1,0 \leq i, j$ $\leq k$ by

$$
E\left(w_{i}(s) w_{j}(t)\right)=\min (s, t) \delta_{i j} .
$$

This suggests that I may be extended to $B$-valued Brownian motion using the methods of II. As it turns out, the resulting Theorem 1 of $\$ 4$ contains both I and II as special cases, and is obtained in a self-contained manner independent of I and II.

2. Some properties of Brownian motion on $B$. Here we provide some basic lemmas. The content of Lemma 2 is found in [7] and can also be expressed in slightly different terms using [6].

Lemma 2. For $(B, \mu)$ as in II, let $\mathcal{L}$ be the closure of $B^{*}$ in $L_{2}(B, \mu)$. For each $L \in \mathcal{L}$ the convergent Bochner integral $x_{L}=\int_{B} L(x) x \mu(d x) \in B$ exists. $H$ 
$=\left\{x_{L}: L \in \mathcal{L}\right\} \subseteq B$ is a real separable Hilbert space isometrically isomorphic to $\mathcal{L}$ under the inner product $\left(x_{L_{1}}, x_{L_{2}}\right)=\int_{B} L_{1}(x) L_{2}(x) \mu(d x)$. On $H,\|\cdot\|_{B} \leq\|\mu\|\|\cdot\|_{H}$ where $\|\mu\|^{2}=\int_{B}\|x\|_{B}^{2} \mu(d x)<\infty$. If $y^{*} \in B^{*}$ and $y=\int_{B} y^{*}(x) x \mu(d x)$, then $(y, x)_{H}=y^{*}(x)$ for every $x \in H$. If $\left\{x_{j}^{*}: j \geq 1\right\} \subseteq B^{*}$ is a complete orthonormal sequence for $\mathcal{L}$ and $\left\{x_{j}: j \geq 1\right\} \subseteq H$ is the set of images $x_{j}=\int_{B} x_{j}^{*}(x) x \mu(d x)$ $(j \geq 1)$, then $\sum_{j=1}^{k} x_{j}^{*}(x) x_{j} \rightarrow x$ as $k \rightarrow \infty$, everywhere on $H$ in the sense of the $H$ norm and almost everywhere on $B$ in the $B$-norm. The closure $\bar{H}$ of $H$ in $B$ is the topological support of $\mu$ on $B$ and if elements of $B$ are interpreted as (evaluation) functions on $B^{*}, H$ may be interpreted as the reproducing kernel Hilbert space of $\mu$.

Proof. That $\boldsymbol{H}$ is separable follows from [6] and the remainder is given in [7].

By [1, Theorems 2 and 3] and the fact that $\|\cdot\|_{B} \leq M\|\cdot\|_{H}$ we have that $\|\cdot\|_{B}$ is a measurable norm in the sense of Gross [2] and hence $(H, \bar{H})$ is an abstract Wiener space.

Lemma 3. Let $B$ be a real separable Banach space with norm $\|\cdot\|_{B}$. Let $H$ be a subspace of $B$ which is a real Hilbert space in the norm $\|\cdot\|_{H}$ and assume $\|\cdot\|_{B}$ is a measurable norm on $H$. Let $K$ denote the unit ball of $H$, i.e. $K=\left\{x \in H:\|x\|_{H}\right.$ $\leq 1$ ). Then $K$ is a compact subset of $B$.

Proof. First we show $K$ is a closed subset of $B$. Let $\left\{y_{n}\right\} \subseteq K$ and assume $\lim _{n} y_{n}=y \in B$ in the norm $\|\cdot\|_{B}$. Now $\left\{y_{n}\right\} \subseteq K$ implies there is a subsequence $\left\{y_{n}\right\}$ such that $\left\{y_{n}\right\}$ converges weakly in $H$ to $z \in H$. Now $\|z\|_{H} \leq 1$ by the uniform boundedness principle, and since $\left\{y_{n}\right\}$ also converges to $y$ in $\|\cdot\|_{B}$ we have $\left\{y_{n}\right\}$ converging weakly to $y$ and to $z$ in $B$ because $B^{*}$ can be viewed as a subset of $H^{*}$. That is, since $\|\cdot\|_{B}$ is a measurable norm on $H$ we have a constant $M$ such that $\|\cdot\|_{B} \leq M\|\cdot\|_{H}$, and hence $B^{*}$ can be continuously injected into $H^{*}$. Now $B^{*}$ separating points of $B$ implies $y=z$, and hence $z \in K$ implies $y \in K$. Hence $K$ is closed in $B$.

Now we show $K$ is compact in $B$. To do this we note that since $\|\cdot\|_{B}$ is a measurable norm on $H$ we can construct a second measurable norm on $H$ as in [2], call it $\|\cdot\|_{1}$, such that for $r>0, V_{r}=\left\{x \in H:\|x\|_{1} \leq r\right\}$ has a compact closure in $B$. Now $\|\cdot\|_{1}$ measurable on $H$ implies there exists an $M>0$ such that $\|x\|_{1} \leq M\|x\|_{H}$ for all $x \in H$, and hence $K \subseteq\left\{x \in H:\|x\|_{1} \leq M\right\}$. Thus $K$ has compact closure in $B$ and since $K$ is closed we have $K$ compact.

There are three separable Banach spaces, each with a mean zero Gaussian measure situated on its Borel subsets, which figure in our analysis. Of these, $\left(C_{B}, P\right)$ and $(B, \mu)$ have already been introduced. The third is $(C, \nu)$ where $C$ is the space of real-valued continuous maps on $[0,1]$ which vanish at zero (with the supremum norm) and $\nu$ is Wiener measure. Each of $\left(C_{B}, P\right),(C, \nu),(B, \mu)$ satisfies the hypotheses of Lemma 2. Let $\& \subseteq C_{B}, H_{0} \subseteq C, H \subseteq B$ denote the respective Hilbert spaces given for each of these spaces by Lemma 2 , and let $\mathscr{K}, K_{0}, K$ be the respective unit balls of these spaces. Then Lemma 3 applies to $\mathscr{K}, K_{0}$ and $K$. 
Using Lemma 2 one may prove the following familiar characterization of $H_{0}: \phi \in H_{0}$ iff $\phi(0)=0, \phi$ is absolutely continuous with respect to Lebesgue measure on $[0,1]$ and $\int_{0}^{1}[(d / d t) \phi(t)]^{2} d t<\infty$. The inner product on $H_{0}$ is

$$
\left(\phi_{1}, \phi_{2}\right)_{H_{0}}=\int_{0}^{1} \frac{d}{d t} \phi_{1}(t) \frac{d}{d t} \phi_{2}(t) d t .
$$

Our next result enables us to interpret $\&$ as a denumerable direct sum of copies of $H_{0}$.

Lemma 4. \& has the following characterization in terms of any set $\left\{x_{j}^{*}: j \geq 1\right\}$ $\subseteq B^{*}$ such that $\left\{x_{j}: j \geq 1\right\}$ is a complete orthonormal set for $H: f \in \mathcal{A}$ iff $f(0)=0, f(t) \in H$ for each $t \in[0,1]$, each $x_{j}^{*}(f) \in H_{0}$, and

$$
\sum_{j} \int_{0}^{1}\left[(d / d t) x_{j}^{*}(f)(t)\right]^{2} d t<\infty
$$

The inner product on $\&$ is given by

$$
\left(f_{1}, f_{2}\right)_{d 4}=\sum_{j} \int_{0}^{1} \frac{d}{d t} x_{j}^{*} f_{1}(t) \frac{d}{d t} x_{j}^{*} f_{2}(t) d t \quad \text { for } f_{1}, f_{2} \in \text { \&. }
$$

In somewhat greater detail we have the following:

(a) $\&=H_{0} \otimes H$ (the tensor product).

(b) If $x^{*} \in B^{*}$ and $f \in \mathcal{A}$ then $x^{*} f \in H_{0}$ and, for every $\phi \in H_{0},\left(x^{*} f, \phi\right)_{H_{0}}$ $=(f, \phi x)_{\text {d, }}$ where $\left(x^{*} f\right)(t)=x^{*}(f(t)), t \in[0,1] .\left\|x^{*} f\right\|_{H_{0}} \leq\|f\|_{\mathcal{A}}\|x\|_{H}$.

(c) If $f \in \mathcal{A}$ and $t \in[0,1]$ then $f(t) \in H$ and, for every $x^{*} \in B^{*},(f(t), x)_{H}$ $=(f, \min (t, \cdot) x)_{\text {d. }}\|f(t)\|_{H} \leq\|f\|_{\text {d }} \sqrt{t}$.

(d) For $\left\{x_{j}^{*}: j \geq 1\right\} \subseteq B^{*}$ and $\left\{x_{j}: j \geq 1\right\} \subseteq H$ as above, $\sum_{j=1}^{k} x_{j}^{*}(f) x_{j} \rightarrow f$ as $k \rightarrow \infty$ everywhere on $\&$ in the sense of $\&$ norm and almost everywhere on $C_{B}$ in the sense of $C_{B}$ norm. That is, if $P$ is the Gaussian measure induced on the Borel subsets of $C_{B}$ by Brownian motion on $B$, then with P-probability one for $f \in C_{B}$

$$
\sup _{0 \leq t \leq 1}\left\|f(t)-\sum_{j=1}^{k} x_{j}^{*}(f(t)) x_{j}\right\|_{B} \rightarrow 0 \text { as } k \rightarrow \infty,
$$

and the law of $x_{j}^{*} f(t)(j \geq 1)$ is that of mutually independent one dimensional Brownian motions normalized as usual.

Proof. For each $t \in[0,1], x^{*} \in B^{*}$, let $\Lambda_{t, x^{*}}(f)=x^{*}(f(t))$ for $f \in C_{B^{*}}$. Then $\Lambda_{t, x^{*}} \in C_{B^{*}}$ and these functionals separate points of $C_{B}$. To prove $\&=H_{0} \otimes H$, suppose $t \in[0,1], x^{*} \in B^{*}$. We first show that the element of $C_{B}$ defined by $\min \{t, \cdot\} x$ is the Bochner integral $\int_{C_{b}} \Lambda_{t, x^{*}}(f) f P(d f)$ and therefore by Lemma 2 applied to $\left(C_{B}, P\right)$ we have $\min (t, \cdot) x \in \mathcal{H}$. We proceed by evaluation of the two expressions. If $s \in[0,1], y^{*} \in B^{*}$, then

$$
\Lambda_{s, y^{\bullet}}(\min (t, \cdot) x)=\min (t, s) y^{*}(x)=\min (t, s)(y, x)_{H}
$$


by Lemma 2 . Now

$$
\begin{aligned}
\Lambda_{s, y^{*}}\left(\int_{C_{b}} \Lambda_{t, x^{*}}(f) f P(d f)\right) & =\int_{C_{\Delta}} x^{*}(f(t)) y^{*}(f(s)) P(d f) \\
& =\int_{C_{\Delta}} x^{*}(f(\min (t, s))) y^{*}(f(\min (t, s))) P(d f)
\end{aligned}
$$

by independence of increments, and using the stationarity of the increments of Brownian motion on $B$ we have (3) equal to

$$
\min (t, s) \int_{C_{B}} x^{*}(f(1)) y^{*}(f(1)) P(d f)=\min (t, s)(y, x)_{H}
$$

since $f \rightarrow f(1)$ induces the measure $\mu=\mu_{1}$ on $B$. Combining (2) and (4) we have $\min (t, \cdot) x \in \mathcal{H}$. From (3) and (4) we have the factorization

$$
\begin{aligned}
(\min (s, \cdot) y, \min (t, \cdot) x)_{d^{4}} & =\min (s, t)(x, y)_{H} \\
& =(\min (s, \cdot), \min (t, \cdot))_{H_{0}}(x, y)_{H} .
\end{aligned}
$$

This proves $\&=H_{0} \otimes H$ provided the elements $\left\{\min (t, \cdot) x: 0 \leq t \leq 1, x^{*}\right.$ $\left.\in B^{*}\right\}$ can be shown to span \& (for a discussion of tensor products of reproducing kernel Hilbert spaces see [9]). To see this, suppose $f_{0} \in \mathcal{\&}$ and $\left(f_{0}, \min (t, \cdot) x\right)_{d}=0$ for all $t \in[0,1], x^{*} \in B^{*}$. By Lemma 2 there is an element $L_{0}$ belonging to the closure of the subspace of $L^{2}\left(C_{B}, P\right)$ spanned by $C_{B}^{*}$ for which $f_{0}=\int_{C_{b}} L_{0}(f) f P(d f)$. Then

$$
\Lambda_{t, x^{*}}\left(f_{0}\right)=\int_{C_{B}} L(f) \Lambda_{t, x^{*}}(f) P(d f)=\left(f_{0}, \min (t, \cdot) x\right)_{d t}=0
$$

for all $t$ and $x^{*}$. Hence $f_{0}=0$ in $C_{B}$ and in $\alpha^{4}$. This completes the proof of $\mathcal{H}=H_{0} \otimes H$.

To prove (b) suppose $x^{*} \in B^{*}, f \in \mathcal{H}, \phi \in H_{0}$. If $f$ is of the specialform $f=\phi_{1} x_{1}$ with $\phi_{1}^{*} \in C^{*}, x_{1}^{*} \in B^{*}$, then $x^{*} f=\phi_{1} x^{*}\left(x_{1}\right) \in H_{0}$ and $(f, \phi x)_{\text {\& }}$ $=\left(\phi_{1} x_{1}, \phi x\right)_{\text {A }}=\left(x_{1}, x\right)_{H}\left(\phi_{1}, \phi\right)_{H_{0}}=x^{*}\left(x_{1}\right)\left(\phi_{1}, \phi\right)_{H_{0}}=\left(x^{*}\left(\phi_{1} x_{1}\right), \phi\right)_{H_{0}}=\left(x^{*} f, \phi\right)_{H_{0}}$. Thus for every $f$ expressible as a finite sum of elements of the form $\phi_{1} x_{1}$ we have $x^{*} f \in H_{0}$ and $\left(x^{*} f, \phi\right)_{H_{0}}=(f, \phi x)$. To extend this to all $f \in \mathcal{A}$ we need for $f$ of the type of sum just considered the inequality $\left\|x^{*} f\right\|_{H_{0}} \leq\|f\|_{\mathcal{A}} \cdot\|x\|_{H}$. To prove this note that if $\left\{\phi_{j}^{*}: j \geq 1\right\} \subseteq C^{*}$ and $\left\{\phi_{j}: j \geq 1\right\} \subseteq H_{0}$ is complete and orthonormal for $H_{0}$ then for $f$ of the above type,

$$
\left\|x^{*} f\right\|_{H_{0}}^{2}=\sum_{j}\left(x^{*} f, \phi_{j}\right)_{H_{0}}^{2}=\sum_{j}\left(f, \phi_{j} x\right)^{2} \leq\|f\|_{d}^{2}\|x\|_{H}^{2}
$$

since $\left\{\phi_{j} x: j \geq 1\right\} \subseteq \&$ are orthogonal in the tensor product and each have norm squared equal to $\|x\|_{H}^{2}$. If $f \in \mathcal{\&}$ then there exists $f_{n} \in \mathcal{H}$ of the above type tending to $f$ in $\mathcal{H}$. Now $\mathcal{A} \subseteq C_{B}$ and $x^{*} \in B^{*}$ implies $x^{*}\left(f_{n}(t)\right) \rightarrow x^{*}(f(t))$ as $n \rightarrow \infty$ since $f_{n} \rightarrow f$ in 4 implies $f_{n} \rightarrow f$ in $C_{B}$ by Lemma 2 . Further, by the above inequality $x^{*} f_{n}$ converges in $H_{0}$ as $n \rightarrow \infty$. Combining the last two statements we have $x^{*} f \in H_{0}$ and $x^{*} f_{n} \rightarrow x^{*} f$ in $H_{0}$. Finally, 


$$
\left(x^{*} f, \phi\right)_{H_{0}}=\lim _{n}\left(x^{*} f_{n}, \phi\right)_{H_{0}}=\lim _{n}\left(f_{n}, x\right)_{d \psi}=(f, \phi x)_{d}
$$

and

$$
\left\|x^{*} f\right\|_{H_{0}}^{2}=\lim _{n}\left\|x^{*} f_{n}\right\|_{H_{0}}^{2} \leq \lim _{n}\left\|f_{n}\right\|_{d H}^{2}\|x\|_{H}^{2}=\|f\|_{d H}^{2}\|x\|_{H}^{2} .
$$

The proof of (c) is analogous to (b). Suppose $f \in \mathcal{H}$ and $t \in[0,1]$. If $f$ is of the form $\phi_{1} x_{1}$ for some $\phi_{1}^{*} \in C^{*}, x_{1}^{*} \in B^{*}$ then $f(t)=\phi_{1}(t) x_{1} \in H$ and if $x \in H$ then from Lemma $2(f(t), x)_{H}=x^{*} f(t)=(f, \min (t, \cdot) x)_{\mathcal{H}}$ from part (a). If $f$ is a finite sum of elements of the above type then $f(t) \in H,(f(t), x)_{H}$ $=(f, \min (t, \cdot x))_{d}$ and

$$
\|f(t)\|_{H}^{2}=\sum_{j}\left(f(t), x_{j}\right)_{H}^{2}=\sum_{j}\left(f, \min (t, \cdot) x_{j}\right)_{\mathcal{H}}^{2} \leq\|f\|_{\mathcal{H}}^{2}\|\min (t, \cdot)\|_{H_{0}}^{2}=t\|f\|_{\mathcal{H}}^{2}
$$

Suppose $f_{n}$ are such finite sums and $f_{n} \rightarrow f$ in f . Then $f_{n}(t)$ converges to $f(t)$ in $B$ and $x^{*} f_{n}(t) \rightarrow x^{*} f(t)$ for each $x^{*} \in B^{*}$. By the previous inequality $f_{n}(t)$ converges in $H$, and hence $f(t) \in H$ and $f_{n}(t) \rightarrow f(t)$ in $H$. By passage to the limit we get $(f(t), x)_{H}=(f, \min (t, \cdot) x)_{\mathcal{H}}$ and $\|f(t)\|_{H} \leq\|f\|_{d} \sqrt{t}$.

To prove (d) assume $\left\{x_{j}^{*}: j \geq 1\right\} \subseteq B^{*}$ and $\left\{x_{j}: j \geq 1\right\} \subseteq H$ is a complete orthonormal set for $H$. Likewise suppose $\left\{\phi_{n}^{*}: n \geq 1\right\} \subseteq C^{*}$ and $\left\{\phi_{n}: n \geq 1\right\}$ $\subseteq H_{0}$ is complete and orthonormal for $H_{0}$. Then from (a) $\left\{\phi_{n} x_{j}: n \geq 1, j \geq 1\right\}$ $\subseteq \mathcal{H}$ is complete and orthonormal for $\mathcal{H}$. For arbitrary $n \geq 1, j \geq 1$ we see that for every $\phi \in H_{0}, x \in H, \phi_{n}^{*} x_{j}^{*}(\phi x)=\phi_{n}^{*}(\phi) x_{j}^{*}(x)$ by linearity. From Lemma 2 we have $\phi_{n}^{*} x_{j}^{*}(\phi x)=\left(\phi_{n}, \phi\right)_{H_{0}}\left(x_{j}, x\right)_{h}$ and hence $\phi_{n}^{*} x_{j}^{*}$ yields $\phi_{n} x_{j}$ by Bochner integration on $C_{B}$. Then everywhere on $\&$ and in $\&$ norm we have $f$ $=\sum_{n, j}\left(f, \phi_{n} x_{j}\right)_{d} \phi_{n} x_{j}=\sum_{n, j} \phi_{n}^{*}\left(x_{j}^{*}(f)\right) \phi_{n} x_{j}$. For each $j \geq 1$ the series may be summed on $n$ in $\&$ obtaining

$$
f=\sum_{j}\left(\sum_{n}\left(f, \phi_{n} x_{j}\right)_{d} \phi_{n}\right) x_{j}=\sum_{j}\left(\sum_{n}\left(x_{j}^{*} f, \phi_{n}\right)_{H_{0}} \phi_{n}\right) x_{j}=\sum_{j} x_{j}^{*}(f) x_{j} .
$$

The argument may be repeated almost everywhere on $C_{B}$ in $C_{B}$ norm and hence $f=\sum_{j} x_{j}^{*}(f) x_{j}$ almost everywhere on $C_{B}$.

Using the explicit description of $H_{0}$ given previous to the present lemma the characterization of $\&$ with which we began the statement of the lemma follows easily from the above series representation.

Since $P$ is a mean zero Gaussian measure on $C_{B}^{1}$ it follows easily from (3) and (4) (since the joint distributions are all Gaussian) that $x_{j}^{*} f(t)(j \geq 1)$ are independent one dimensional Brownian motions.

For every $\epsilon>0$ let $\mathfrak{K}_{e}$ denote the open $\epsilon$-neighborhood of $\mathscr{K}$ in $C_{B}$.

Lemma 5. For each $\epsilon>0$, there exists $r>1$ such that

$$
P\left\{f \in C_{B}: f / \sqrt{2 \log \log s} \notin \mathscr{K}_{\epsilon}\right\} \leq \exp \left(-r^{2} \log \log s\right)
$$

for all sufficiently large s. 
Proof. This result can be proved just as Proposition 1 of [8] is obtained.

Lemma 6. If $\epsilon>0$ one may choose $c>1$ sufficiently close to one so that for every $f \in \Omega_{B}$ the statements $\left[c^{n}\right] \leq s \leq\left[c^{n+1}\right]$ and $f\left(\left[c^{n+1}\right] \cdot\right) /\left\{2\left[c^{n+1}\right] \log \log \left[c^{n+1}\right]\right\}^{1 / 2}$ $\in \mathscr{K}_{e}$ together imply $f(s \cdot) / \sqrt{2 s \log \log s} \in \mathscr{K}_{2 e}$ for all sufficiently large $n$.

Proof. Suppose $\epsilon>0$ and choose $c>1$ so that for all sufficiently large $n$, $\gamma_{n} \epsilon+\left(\gamma_{n}-1\right)\|P\|<2 \epsilon$ where

$$
\gamma_{n}=\left(\frac{\left[c^{n+1}\right] \log \log \left[c^{n+1}\right]}{\left[c^{n}\right] \log \log \left[c^{n}\right]}\right)^{1 / 2} .
$$

This is possible because $\left[c^{n+1}\right]<c^{2}\left[c^{n}\right]$ for all large $n$. If $h \in \mathscr{K}, f \in C_{B}$,

$$
\left\|f\left(\left[c^{n+1}\right] \cdot\right) /\left(2\left[c^{n+1}\right] \log \log \left[c^{n+1}\right]\right)^{1 / 2}-h(\cdot)\right\|_{c_{0}}<\epsilon,
$$

then

$$
\left\|f(s \cdot) /\left(2\left[c^{n+1}\right] \log \log \left[c^{n+1}\right]\right)^{1 / 2}-h\left(\frac{s \cdot}{\left[c^{n+1}\right]}\right)\right\|_{c_{0}}<\epsilon
$$

and

$$
\left\|h\left(\frac{s \cdot}{\left[c^{n+1}\right]}\right)\right\|_{\alpha} \leq \frac{s}{\left[c^{n+1}\right]}\|h\|_{\alpha} \leq 1 .
$$

Hence $h\left(\left(s /\left[c^{n+1}\right]\right) \cdot\right) \in \mathscr{K}$ and

$$
\begin{aligned}
& \left\|f(s \cdot) /(2 s \log \log s)^{1 / 2}-h\left(\frac{s \cdot}{\left[c^{n+1}\right]}\right)\right\|_{C_{s}} \\
& \leq\left\|f(s \cdot) /(2 s \log \log s)^{1 / 2}-\left(\frac{\left[c^{n+1}\right] \log \log \left[c^{n+1}\right]}{s \log \log s}\right)^{1 / 2} h\left(\frac{s \cdot}{\left[c^{n+1}\right]}\right)\right\|_{C_{s}} \\
& \quad+\|P\|\left\|\left(\frac{\left[c^{n+1}\right] \log \log \left[c^{n+1}\right]}{s \log \log s}\right)^{1 / 2} h\left(\frac{s \cdot}{\left[c^{n+1}\right]}\right)-h\left(\frac{s \cdot}{\left[c^{n+1}\right]}\right)\right\|_{d} \\
& \leq \gamma_{n} \epsilon+\left(\gamma_{n}-1\right)\|P\|<2 \epsilon
\end{aligned}
$$

if $n$ is sufficiently large.

For what follows we assume $\left\{x_{j}^{*}: j \geq 1\right\} \subseteq B^{*}$ and $\left\{x_{j}: j \geq 1\right\} \subseteq H$ is complete and orthonormal for $H$. For each $k \geq 1$ and $f \in C_{B}$ let $f^{(k)}$ $=\sum_{j=1}^{k} x_{j}^{*}(f) x_{j}$.

Lemma 7. For each $\epsilon>0$ and $r>1$ there exists $k$ sufficiently large so that

$$
P\left(f \in C_{B}:\left\|f-f^{(k)}\right\|_{C_{B}} \geq \epsilon \sqrt{2 \log \log s}\right) \leq \exp \left(-r^{2} \log \log s\right)
$$

for all sufficiently large s. 
Proof. By (d) of Lemma 4 this result follows just as in Lemma 4 of [8].

The main theorem and some corollaries. Our basic theorem is the following and implies the result of Strassen mentioned in I and that of LePage in II.

Theorem 1. Let $\{W(t): 0 \leq t<\infty\}$ be Brownian motion on $B$ and for each $t \in[0,1], s \geq 3$, let

$$
\zeta_{s}(t)=W(s t) / \sqrt{2 s \log \log s} .
$$

Then the net $\left\{\zeta_{s}: s \geq 3\right\}$ is a subset of $C_{B}$ and with probability one converges in $C_{B}$ to the compact set $\mathscr{K}$ and clusters at every point of $\mathscr{K}$, where $\mathcal{K}$ is the unit ball of the reproducing kernel Hilbert space (equivalently, $\mathscr{K}$ is the unit ball of the Hilbert subspace of $C_{B}$ which generates $P$ ).

Proof. That $\mathscr{K}$ is compact in $C_{B}$ follows from Lemma 3 by applying the lemma to $C_{B}, \mathcal{H}$, and $\|\cdot\|_{C_{B}}$. For every $\epsilon>0$, there exists $r>1$ such that

$$
\begin{aligned}
\operatorname{Pr}\left(\zeta_{s} \notin \mathscr{K}_{\epsilon}\right) & =P\left(f \in C_{B}:\|f-\mathscr{K}\|_{C_{B}} \geq \epsilon \sqrt{2 \log \log s}\right) \\
& \leq \exp \left(-r^{2} \log \log s\right)
\end{aligned}
$$

for all sufficiently large $s$ by Lemma 5 . Hence by the Borel-Cantelli lemma for $c>1$ there is a set $A$ of probability one such that the sequence $\zeta_{\left[c^{n}\right]} \in \mathscr{K}_{\mathrm{e}}$ for all but finitely many $n$. Therefore by Lemma $6 \zeta_{s} \in \mathscr{K}_{2 e}$ for all $s$ sufficiently large on the set $A$. Letting $\epsilon$ converge to zero through a countable set we have

$$
\operatorname{Pr}\left\{\zeta_{s} \rightarrow \mathscr{K} \text { as } s \rightarrow \infty \text { in } C_{B}\right\}=1 \text {. }
$$

To prove $\mathscr{K}$ is almost surely the set of cluster points of $\left\{\zeta_{s}: s \geq 3\right\}$ it suffices by the separability of $\mathscr{K}$ to prove that if $h \in \mathcal{K},\|h\|_{\alpha}<1$ and $\epsilon>0$ there is a $c>1$ so that with probability one $\left\|\zeta_{\left[c^{n}\right]}-h\right\|_{c_{\varepsilon}}<\epsilon$ for infinitely many $n$. By Lemma 7 choose $r>1$ and $k$ sufficiently large so that (5) holds with $\epsilon$ replaced by $\epsilon / 3$ for all sufficiently large $s$. By Lemma $4(d)$ choose $k$ large enough so that $\left\|h-h^{(k)}\right\|_{c_{B}}<\epsilon / 3$. Then for every $c>1$, applying these estimates and the Borel-Cantelli lemma, we have with probability one that

$$
\begin{aligned}
& \left\|\zeta_{\left[c^{n}\right]}-h\right\|_{C_{b}} \leq 2 \epsilon / 3+\left\|\zeta_{\left\{c^{n}\right]}^{(k)}-h^{(k)}\right\|_{C_{B}} \\
& \leq 2 \epsilon / 3+\|P\| \sup _{0 \leq t \leq 1}\left\|\zeta_{\left[c^{n}\right]}^{(k)}(t)-h^{(k)}(t)\right\|_{H}
\end{aligned}
$$

for all sufficiently large $n$.

It now suffices to show that with probability one

$$
\sup _{0 \leq t \leq 1}\left\|\zeta_{\left\{c^{n}\right]}^{(k)}(t)-h^{(k)}(t)\right\|_{H}<\epsilon / 3\|P\|
$$

for infinitely many $n$. Our argument follows an idea due to Strassen. 
Let $m \geq 2$ be an integer, $0<\delta<1$, and assume $Z_{j,\left[c^{n}\right]}$ and $h_{j}(j=1, \ldots, k)$ are the $j$ th-coordinates of $\zeta_{\left[c^{n}\right]}^{(k)}$ and $h^{(k)}$. We define the event

$$
\begin{aligned}
A_{n}=\left\{w:\left|\left(Z_{\left.j, k c^{n}\right]}(w, i / m)-Z_{j,\left[c^{n}\right]}(w, i-1 / m)\right)-\left(h_{j}(i / m)-h_{j}(i-1 / m)\right)\right|\right. \\
<\delta / k \text { for } i=2, \ldots, m \text { and } j=1, \ldots, k\} .
\end{aligned}
$$

Then

$$
\operatorname{Pr}\left(A_{n}\right) \geq \prod_{i=2}^{m} \prod_{j=1}^{k} \frac{1}{\sqrt{2 \pi}} \int_{a_{v}}^{b_{v}} e^{-s^{2} / 2} d s
$$

where (letting LL denote $\log \log$ )

$$
\begin{aligned}
& a_{i j}=\left|h_{j}(i / m)-h_{j}(i-1 / m)\right| \sqrt{2 m L L\left[c^{n}\right]}, \\
& b_{i j}=\left(\left|h_{j}(i / m)-h_{j}(i-1 / m)\right|+\delta / k\right) \sqrt{2 m L L\left[c^{n}\right]},
\end{aligned}
$$

for $i=2, \ldots, m ; j=1, \ldots, k$. Using the estimate

$$
\int_{a}^{b} \exp \left(-s^{2} / 2\right) d s \geq \frac{\exp \left(-a^{2} / 2\right)}{b}\left(1-\exp \left(-\left(b^{2}-a^{2}\right)\right) / 2\right) \text { for } 0 \leq a<b
$$

we have a constant $\gamma>0$ such that

$$
\operatorname{Pr}\left(A_{n}\right) \geq \gamma \prod_{i=2}^{m} \prod_{j=1}^{k} \frac{\exp \left(-a_{i j}^{2} / 2\right)}{b_{i j}}
$$

for all $n$ sufficiently large (because $0 \leq a_{i j}<b_{i j}$ implies $b_{i j}^{2}-a_{i j}^{2} \geq\left(b_{i j}-a_{i j}\right)^{2}$ $\left.\geq\left(\delta^{2} / k^{2}\right) 2 m L L\left[c^{n}\right]\right)$. Hence there is a constant $\gamma_{1}>0$,

$$
\begin{aligned}
\operatorname{Pr}\left(A_{n}\right) & \geq \frac{\gamma_{1} \exp \left\{\sum_{i=2}^{m} \sum_{j=1}^{k}\left(h_{j}(i / m)-h_{j}(i-1 / m)\right)^{2} m L L\left[c^{n}\right]\right\}}{\left(2 m L L\left[c^{n}\right]\right)^{(m-1) k / 2}} \\
& \geq \frac{\gamma_{1} \exp \left\{-\left\|h^{k}\right\|_{d^{4}} \cdot \mathrm{LL}\left[c^{n}\right]\right\}}{\left(2 m L L\left[c^{n}\right]\right)^{m k / 2}},
\end{aligned}
$$

and since $\theta=\left\|h^{k}\right\|_{\mathcal{H}}<1$ we have

$$
\operatorname{Pr}\left(A_{n}\right) \geq \frac{\gamma_{1}}{\left(\log \left[c^{n}\right]\right)^{\theta}\left(2 m L L\left[c^{n}\right]\right)^{m k / 2}}
$$

Hence for $c=m$ we have $A_{1}, A_{2}, \ldots$ independent and

$$
\operatorname{Pr}\left(A_{n}\right) \geq \frac{\gamma_{1}}{(n \log m)^{\theta}(2 m(\log n+\mathrm{LL} m))^{m k / 2}} \geq \frac{\gamma_{2}}{n^{\theta}(\log n)^{m k / 2}}
$$

for all $n$ sufficiently large.

Now $\theta<1$ implies $\sum_{n=1}^{\infty} \operatorname{Pr}\left(A_{n}\right)=\infty$ so by Borel-Cantelli $\operatorname{Pr}\left(\lim \sup _{n} A_{n}\right)$ $=1$. 
Using the fact that the $H$-norm and the $B$-norm are equivalent on the finite dimensional subspace of $B$ generated by $\left\{x_{1}, \ldots, x_{k}\right\}$ we have by the first part of the proof that with probability one $\zeta_{\left[c^{n}\right]^{(k)}}(t)$ is eventually within $\delta$ of $\mathscr{T} \mathcal{K}^{(k)}$. Here $\mathcal{K}^{(k)}$ is the subset of $\mathscr{K}$ consisting of functions of the form $\sum_{j=1}^{k} x_{j}^{*}(w(t)) x_{j}$. Hence with probability one

$$
\left\|\zeta_{\left[c^{n}\right]}^{(k)}(s)-\zeta_{\left[c^{n}\right]}^{(k)}(t)\right\|_{H} \leq \sqrt{|t-s|}+\delta
$$

for all $0 \leq s, t \leq 1$ and all $n$ sufficiently large. Now if $y \in C_{B}[0,1]$ and $y$ satisfies

(a) $\left\|y^{(k)}(t)-y^{(k)}(s)\right\|_{H} \leq \sqrt{|t-s|}+\delta(0 \leq s, t \leq 1)$,

(b) $\left|\left(y_{j}(i / m)-y_{j}((i-1) / m)\right)-\left(h_{j}(i / m)-h_{j}((i-1) / m)\right)\right|<\delta / k$

for all $j=1, \ldots, k, 2 \leq i \leq m$ where $y_{j}$ is the $j$ th coordinate of $y$, then

$$
\sup _{0 \leq t \leq 1}\left\|y^{(k)}(t)-h^{k}(t)\right\|_{H}<\epsilon / 3\|P\|
$$

provided $m$ is sufficiently large and $\delta$ is sufficiently small. Using the definition of $A_{n}$ and (7) we see that with probability one (6) holds for infinitely many $n$. This concludes the proof.

The next corollary follows immediately from Theorem 1 .

Corollary 1. Let $\theta$ be a continuous function on $C_{B}$ into a Hausdorff topological space $Y$ and assume the notation of Theorem 1. Then with probability one $\left\{\theta \circ \zeta_{s}: s \geq 3\right\}$ converges to the compact set $\theta(\mathscr{K})$ and clusters at each point of $\theta(\mathscr{K})$.

Corollary 2. If $\{W(t): 0 \leq t<\infty\}$ is Brownian motion on $B$, then

$$
\operatorname{Pr}\left(\varlimsup_{s \rightarrow \infty} \frac{\|W(s)\|_{B}}{\sqrt{2 s \log \log s}}=\sup _{x \in K}\|x\|_{B}\right)=1 .
$$

Proof. Since $\|W(s)\|_{B} / \sqrt{2 s \log \log s}=\left\|\zeta_{s}(1)\right\|_{B}$ this result follows from Corollary 1 with $\theta(f)=\|f(1)\|_{B}$ and by showing that $\sup _{f \in \mathscr{X}}\|f(1)\|_{B}=\sup _{x \in K}\|x\|_{B}$. Now if $f \in \mathcal{K}$, then by Lemma $4(\mathrm{c})\|f(1)\|_{H} \leq\|f\|_{\mathcal{H}} \leq 1$ and hence $f(1) \in K$ $\subseteq H$. On the other hand, if $x \in K$ we can set $f(t)=t x$ and then $\|f\|_{A}^{2}=(x, x)_{H}$ $\leq 1$. Hence $f(t)=t x \in \mathscr{K}$ and $\theta(f)=\|x\|_{B}$. By combining the above we have

$$
\sup _{f \in \mathscr{K}}\|f(1)\|_{B}=\sup _{x \in K}\|x\|_{B}
$$

and the proof is complete.

For the following recall the statements I and II of the introduction.

Corollary 3. I holds.

Proof. If $B=\mathbf{R}^{k}$ then $H=B, \mathscr{K}=K_{k}$ and the result in I follows immediately. 
Corollary 4. II holds.

Proof. Construct Brownian motion in $B$, call it $\{W(t): 0 \leq t<\infty\}$, such that $\mu_{1}=\mu$. Let $\theta(f)=f(1)$ for $f \in C_{B}$. Using the stationary independent increments of $\{W(t)\}$ it follows that the joint distributions of $\left\{\xi_{n}: n \geq 3\right\}$ are identical to those of $\left\{\theta\left(\zeta_{n}\right): n \geq 3\right\}$ where $\zeta_{n}$ is as in Theorem 1. Hence with probability one $\left\{\xi_{n}: n \geq 3\right\}$ converges to the set $\theta(\mathscr{K})$ and clusters at each point of $\theta(\mathscr{K})$ by Corollary 1. Now by the argument given in Corollary $2 \theta(\mathscr{K})=K$ and hence the proof is complete.

Remark. In view of Lemma 3 it follows that $K$ is compact in $B$ and hence Corollary 4 actually implies the sequence $\left\{\zeta_{n}: n \geq 3\right\}$ is relatively norm compact with probability one and that its limit points consist precisely of $K$ (with probability one). This is slightly stronger than II. Finally II generalizes the law of the iterated logarithm of [3] to Gaussian random variables in $B$.

\section{REFERENCES}

1. R. Dudley, J. Feldman and L. LeCam, On seminorms and probabilities, and abstract Wiener spaces, Ann. of Math.

2. L. Gross, Lectures in modern analysis and applications. II, Lecture Notes in Math., vol. 140, Springer-Verlag, New York.

3. P. Hartman and A. Wintner, On the law of the iterated logarithm, Amer. J. Math. 63 (1941), 169-176. MR 2,228.

4. W. Feller, An introduction to probability theory and its applications. Vol. II, Wiley, New York, 1966. MR 35 \# 1048.

5. G. Kallianpur, Abstract Wiener processes and their reproducing kernel Hilbert spaces, $Z$. Wahrscheinlichkeitstheorie und Verw. Gebiete 17 (1971), 113-123. MR 43 \#6961.

6. J. Kuelbs, Expansions of vectors in a Banach space related to Gaussian measures, Proc. Amer. Math. Soc. 27 (1971), 364-370. MR 42 \#2517.

7. R. LePage, Note relating Bochner integrals and reproducing kernels to series expansions on a Gaussian Banach space, Proc. Amer. Math. Soc. 31 (1972),

8. , Log Log law for Gaussian processes, Z. Wahrscheinlichkeitstheorie und Verw. Gebiete (to appear).

9. E. Parzen, Probability density functionals and reproducing kernel Hilbert spaces, Proc. Sympos. Time Series Analysis (Brown University, 1962), Wiley, New York, 1963, pp. 155-169. MR 26 \#7119.

10. V. Strassen, An invariance principle for the law of the iterated logarithm, $\mathrm{Z}$. Wahrscheinlichkeitstheorie und Verw. Gebiete 3 (1964), 211-226. MR 30 \#5379.

Department of Mathematics, University of Wisconsin, Madison, Wisconsin 53706 (Current address of J. Kuelbs)

Department of Mathematics, Universtty of Colorado, Boulder, Colorado 80302

Current address (R. LePage): Department of Probability and Statistics, Michigan State University, East Lansing, Michigan 48823 